\title{
Veinte años de políticas de evaluación general del sistema educativo en España
}

Núm. 2 (2014), pp. 01-21.

Alejandro Tiana Ferrer ${ }^{1}$

Recibido: Febrero, 2014

Aceptado: Marzo, 2014

JEL Clasif: JEL-class: I20, I21, I28

${ }^{1}$ Facultad de Educación. UNED. C/ Juan del Rosal, 14, 28040 Madrid. $\underline{\text { atiana@edu.uned.es }}$ 


\begin{abstract}
In the last two decades a system for evaluating the education system has been designed, organized and developed in Spain. Evaluation policies developed in that period have adopted different orientations in relation to the aims, approaches and design of the evaluation projects set in place. In this paper the three phases which may be differentiated in that process are identified and characterized, paying a special attention to their entity and characteristics. The analysis of such phases, each one of them crossing different governments and translated into different norms and regulations, has an evident interest, especially when put in connection with the broader educational policies in which they are inserted. The main element allowing to distinguish each one of these three phases consists in the mission assigned to evaluation, from which their characteristics and instrumentation derive.
\end{abstract}

Key Words: educational evaluation, Spanish Education system, educational policy, outcomes evaluation, educational indicators, international evaluation studies

\title{
Resumen
}

Las últimas dos décadas han asistido al diseño, organización y desarrollo de un sistema de evaluación general del sistema educativo en España. Las políticas de evaluación desarrolladas en este tiempo han adoptado distintas orientaciones, tanto en lo que se refiere a los fines de la evaluación emprendida como al enfoque y diseño de los estudios realizados. En este artículo se identifican y caracterizan las tres etapas que pueden distinguirse en ese proceso, cada una de las cuales tiene entidad propia y características específicas. El análisis de dichas etapas, que cruzan gobiernos de distinto signo político y se reflejan en leyes y normativas diferentes, tiene un interés indudable, sobre todo si se ponen en conexión con las políticas educativas más amplias en las que se insertan. El elemento fundamental para distinguir unas de otras consiste en la misión asignada a la evaluación, de la que derivan sus características e instrumentación.

Palabras clave: evaluación educativa, sistema educativo español, política de la educación, evaluación del rendimiento, indicadores de la educación, estudios internacionales de evaluación 


\section{Introducción}

En el momento de escribir estas páginas se cumplen muy aproximadamente veinte años desde que comenzó a desarrollar sus trabajos el Instituto Nacional de Calidad y Evaluación (INCE), el primer organismo creado en España con la función específica de realizar la evaluación general del sistema educativo. El INCE fue creado en 1990 por la Ley Orgánica de Ordenación General del Sistema Educativo (LOGSE), que le dedicó el artículo 62, incluido en el título cuarto, dedicado a la calidad de la enseñanza. En junio de 1993 se aprobó un real decreto que vino a regular su composición, funciones y organización (RD 928/1993). Casi un año más tarde, el 17 de mayo de 1994, se celebró la reunión constitutiva de su Consejo Rector, en la que se discutieron y aprobaron el marco y las líneas generales que habían de regir la organización y el funcionamiento del Instituto, así como sus primeros proyectos y sus mecanismos de coordinación. Fue así como comenzó la andadura de un organismo que, con varios cambios de denominación y orientación, continúa activo y pujante en la actualidad.

A lo largo de estas dos décadas se han registrado avances importantes en la evaluación general del sistema educativo español, llevándose a cabo un amplio conjunto de iniciativas y emprendiéndose numerosos estudios. Pero es necesario subrayar que las políticas de evaluación desarrolladas en este tiempo han adoptado distintas orientaciones, tanto en lo que se refiere a los fines de la evaluación emprendida como al enfoque y diseño de los estudios realizados.

El propósito de este artículo no es otro que el de identificar y caracterizar las principales etapas que pueden distinguirse en el proceso de construcción y desarrollo de una política de evaluación general del sistema educativo en España. A mi juicio, son tres las fases que cabe distinguir, cada una de las cuales tiene entidad propia y características específicas. El análisis de dichas etapas, que cruzan gobiernos de distinto signo político y se reflejan en leyes y normativas diferentes, tiene un interés indudable, sobre todo si se ponen en conexión con las políticas educativas más amplias en las que se insertan. El elemento fundamental para distinguir unas de otras consiste en la misión asignada a la evaluación, de la que derivan sus características e instrumentación. Espero saber presentar al lector la evolución registrada y las claves para interpretarla. 


\section{La etapa preliminar}

Aunque la creación del INCE representó un hito fundamental en el proceso de construcción de una política de evaluación general del sistema educativo, no constituyó realmente su punto de partida. De hecho, en la década anterior se habían producido varias iniciativas importantes y novedosas en España, que es necesario recordar. En efecto, hay que destacar que el interés por el desarrollo de la evaluación educativa, entendida como una actividad no solamente aplicable a los estudiantes y a sus aprendizajes, sino también a los centros docentes y al conjunto del sistema educativo, apareció ya en algunos de los principales textos normativos y en las diversas propuestas de reforma educativa de los años ochenta y noventa.

Uno de los antecedentes más remotos de esta nueva tendencia se encuentra en el Libro Blanco de 1969, que sirvió de fundamento para la elaboración de la Ley General de Educación (LGE) de 1970. Si bien ese texto no hacía ninguna mención expresa a la evaluación general del sistema educativo, entendida como una tarea que debía incluir varias dimensiones, planteaba ya la necesidad de abordar la evaluación del rendimiento educativo, de la que afirmaba que "se referirá tanto a la acción de los Centros docentes como a los alumnos" (MEC, 1969: 237). Se trataba de un planteamiento sin duda avanzado para su tiempo, sobre todo en lo que respecta a la previsión de evaluación de los centros docentes, que todavía tardaría tiempo en convertirse en realidad. No obstante, conviene señalar que el concepto de evaluación educativa, que apenas comenzaba a incorporarse al lenguaje pedagógico español, se identificaba en la ley sobre todo con la evaluación de los estudiantes, mientras que apenas se esbozaban otras modalidades.

La idea de que resulta necesario evaluar la situación, el estado y las transformaciones del sistema educativo y de sus principales componentes comenzó a extenderse en los años ochenta, paralelamente al desarrollo de las reformas experimentales de las Enseñanzas Medias y de la EGB que emprendió el gobierno socialista tras su victoria electoral de 1982 (Menor y Moreiro, 2010). El Centro de Investigación y Documentación Educativa (CIDE) del Ministerio de Educación y Ciencia puso en marcha el programa denominado de Evaluación externa de la reforma experimental de las Enseñanzas Medias y del ciclo superior de EGB, con el propósito de realizar una evaluación sistemática del proceso de reforma y de sus consecuencias, que 
dio lugar a varios informes públicos (CIDE, 1988, 1990 y 1992). También algunas comunidades autónomas desarrollaron programas similares, como es el caso de la evaluación de la reforma del ciclo superior de EGB realizada en Andalucía (Pérez y Gimeno, 1994). Esas evaluaciones han sido objeto recientemente de una cuidadosa metaevaluación, que nos permite ponerlas en perspectiva, valorando sus contribuciones para la puesta en marcha de la evaluación general del sistema educativo y señalando sus logros y sus limitaciones (García Sánchez, 2011).

Los planteamientos que se difundieron a comienzos de los años ochenta y dieron lugar a esas iniciativas pioneras encontraron un claro reflejo en el Proyecto para la reforma de la enseñanza que presentó para debate en 1987 el Ministerio de Educación y Ciencia. En ese documento se insistía claramente en la necesidad de evaluar el sistema educativo, en sus diferentes ámbitos y modalidades. El texto era categórico cuando afirmaba: "Es más, cualquier reforma de la ordenación educativa, así como cualquier propuesta pedagógicamente innovadora de renovación, tanto de la organización escolar como de los procesos educativos, ha de ir acompañada de un diseño de los procedimientos para su evaluación” (MEC, 1987: 148). Esa idea era reforzada más adelante, insistiendo en que "para establecer y llevar a cabo una política de reforma y de renovación de la enseñanza, es absolutamente necesario diseñar y desarrollar evaluaciones del sistema educativo, en sus diversas modalidades, etapas y áreas, que sean verdaderas investigaciones sobre su funcionamiento efectivo" (MEC, 1987: 150). Se esbozaba así una primera caracterización de qué debería entenderse por una evaluación general del sistema educativo.

Con la orientación planteada en ese proyecto y aprovechando el impulso que representó el examen de la política educativa española llevado a cabo en 1986 por la OCDE (MEC, 1986), se decidió la participación española en el International Assessment of Educational Progress (IAEP), promovido por el National Center for Educational Statistics de Estados Unidos, y en el Reading Literacy Study, impulsado por la International Association for the Evaluation of Educational Achievement (IEA). La realización de estos proyectos exigió la constitución de un equipo dedicado a la evaluación del sistema educativo en el Centro de Investigación y Documentación Educativa, que amplió así sus funciones. Fruto de ese desarrollo fue el cambio de nombre del CIDE en 1989, que pasaría significativamente a denominarse Centro de Investigación, Documentación y Evaluación, aunque conservando sus siglas distintivas. 
Los planteamientos apenas esbozados en el proyecto de reforma de 1987 fueron recogidos y ampliados en 1989 en el Libro Blanco que elaboró el Ministerio de Educación y Ciencia para preparar la reforma educativa de 1990 (MEC, 1989). El documento supuso un claro avance en lo que se refiere a la evaluación del sistema educativo y planteó varias razones para llevarla a cabo. Algunas eran de carácter genérico, como la necesidad de conocer las condiciones del sistema con vistas a tomar decisiones sobre su ordenación o gestión, la exigencia de velar por el cumplimiento de los objetivos de cada nivel y ciclo educativo o el derecho de la sociedad a conocer el funcionamiento del sistema educativo. Un argumento que comenzaba a cobrar importancia, sobre todo tras la adhesión de España a la Comunidad Económica Europea, consistía en la necesidad de aumentar la interrelación de los sistemas educativos nacionales y de garantizar la validez de las acreditaciones de los diversos países, a lo que debía contribuir la realización de evaluaciones comparativas internacionales. Pero el argumento más interesante de entre los expuestos, y que serviría de orientación fundamental para la etapa que entonces se inauguraba, se refería a las implicaciones derivadas del proceso de descentralización de la enseñanza, consecuencia de la nueva organización autonómica del Estado establecida en la Constitución de 1978.

Para los redactores del Libro Blanco, la descentralización tenía como corolario inevitable un aumento de la autonomía curricular de las comunidades autónomas y de los centros docentes, que era valorada positivamente, ya que conduciría a un sistema más diverso, más rico en alternativas y mejor adaptado a las diferentes condiciones sociales y culturales, si bien no se obviaba el riesgo que esa situación podía implicar para la coherencia del sistema ni se minusvaloraba la necesidad de evitar posibles desequilibrios y desigualdades. En las nuevas condiciones, resultaba imprescindible establecer mecanismos para evaluar el conjunto del sistema educativo, respetando al mismo tiempo las competencias de las comunidades autónomas, con el objetivo de asegurar un cierto equilibrio de condiciones educativas a todos los ciudadanos españoles. Esa idea inspiraría claramente la creación y constitución del Instituto Nacional de Calidad y Evaluación en la LOGSE y la orientación de sus trabajos.

Se puede afirmar, sin temor a equivocarse, que el Libro Blanco de 1989 presentó la primera propuesta completa para la evaluación general del sistema educativo que se elaboró en España, entendiendo la evaluación como un conjunto de actividades 
relacionadas entre sí y referidas a una diversidad de ámbitos. Ese documento delimitó claramente el lugar y las características de la evaluación de los estudiantes, de los centros y del sistema educativo, previó su organización institucional en un marco respetuoso de las competencias de las comunidades autónomas y estableció sus principales objetivos y modos de actuación. Fue así como se inauguró la primera etapa de las tres que he anunciado más arriba.

\section{La constitución y puesta en marcha de un sistema de evaluación general del sistema educativo}

Como se dijo al comienzo, la LOGSE creó el Instituto Nacional de Calidad y Evaluación (INCE), en cuyo gobierno y funcionamiento deberían participar las Administraciones educativas y cuyas funciones serían básicamente dos: a) elaborar sistemas de evaluación para las diferentes enseñanzas reguladas en la ley y sus centros; y b) realizar investigaciones, estudios y evaluaciones del sistema educativo, así como proponer iniciativas y sugerencias para la mejora de la calidad de la enseñanza (artículo 62). En desarrollo de esa disposición, el INCE fue regulado por el Real Decreto 928/1993, de 18 de junio (BOE del 6 de julio). En diciembre de ese mismo año, la Conferencia sectorial de Educación dio su aprobación al nombramiento del primer director y por esas fechas comenzó la dotación de personal del centro y se elaboraron sus primeros presupuestos. A mediados de 1994, el INCE estaba plenamente operativo. Comenzaba así la primera etapa de desarrollo de la evaluación general del sistema educativo español, caracterizada por la puesta en marcha de un modelo de evaluación adaptado a la estructura descentralizada del Estado y a la distribución de competencias que en materia de educación habían establecido la Constitución de 1978 y los diversos estatutos de autonomía².

En efecto, la creación del INCE no impedía reconocer que las comunidades autónomas eran las competentes para evaluar el sistema educativo en su ámbito correspondiente. De acuerdo con ese planteamiento general, al nuevo instituto se le

\footnotetext{
${ }^{2}$ Aunque no sea objeto de análisis en estas páginas, cabe recordar que en este mismo periodo comenzó a desarrollarse la evaluación de la calidad de las universidades, de cuya primera etapa puede encontrarse una síntesis valiosa en un libro publicado por la Agencia Nacional de Evaluación de la Calidad y la Acreditación - ANECA (Varios autores, 2007).
} 
asignaron dos conjuntos de funciones, que definieron con nitidez su campo específico de actuación. El primer grupo derivaba de las competencias del Estado en materia de educación, mientras que el segundo planteaba diversos mecanismos de colaboración en las tareas de evaluación emprendidas por las comunidades autónomas. Así, las principales funciones asignadas al INCE fueron las siguientes:

a) Evaluar el grado de adquisición de las enseñanzas mínimas establecidas para los diversos niveles, etapas, ciclos y grados del sistema educativo, en el marco del currículo establecido por las Administraciones educativas y de su desarrollo en los centros docentes. Dicho con otras palabras, los proyectos de evaluación del sistema educativo deberían respetar el modelo de desarrollo curricular que la ley definía.

b) Evaluar las reformas del sistema educativo, así como la estructura, el alcance y los resultados de las innovaciones introducidas, con el propósito de realizar el adecuado seguimiento del sistema educativo, ofreciendo información fiable acerca de su evolución y desarrollo.

c) Elaborar un sistema estatal de indicadores del sistema educativo, similar a los desarrollados por otros países, con la intención de conocer mejor su situación y sus logros y evaluar su grado de eficacia y eficiencia.

d) Coordinar la participación española en los estudios internacionales de evaluación, actividad que aumentaría notablemente durante los años noventa.

e) Cooperar con otras Administraciones en la elaboración de sistemas de evaluación para las diferentes enseñanzas y los centros docentes, informar a los distintos sectores de la sociedad acerca del funcionamiento y los resultados del sistema educativo, proporcionar e intercambiar información que facilitase la toma de decisiones y publicar y difundir los resultados de las evaluaciones realizadas.

De acuerdo con estas previsiones, el INCE fue concebido como un organismo estatal, aunque en un marco de estrecha cooperación con las comunidades autónomas, quienes participaron en su organización y funcionamiento a través de su Consejo Rector y de los informes de los estudios llevados a cabo, que debían presentarse a la 
Conferencia sectorial de Educación. No obstante, es necesario destacar que el hecho de no haber sido configurado como un organismo independiente del Ministerio de Educación y Ciencia fue objeto de algunas críticas. Con objeto de reforzar su carácter técnico y su autonomía, se creó un Comité Científico, formado por personas de reconocido prestigio académico y profesional y responsable de supervisar el diseño y aplicación de los trabajos a realizar.

Paralelamente a la puesta en marcha del INCE, algunas comunidades autónomas crearon sus propios centros de evaluación, siendo pioneros el Consell Superior d'Avaluació del Sistema Educatiu de Cataluña, creado en 1993, y el Instituto Canario de Calidad y Evaluación (ICEC), creado en 1995. De ese modo, en los años noventa se fue configurando una red de organismos autonómicos encargados de la evaluación del sistema educativo, que cooperaban entre sí, al tiempo que desarrollaban sus propios proyectos. En conjunto, puede decirse que ese fue el inicio de un interesante conjunto de iniciativas de evaluación general del sistema educativo español, que serán comentadas más adelante.

En los años posteriores continuó la evolución paulatina de ese modelo inicial. La Ley Orgánica de la Participación, la Evaluación y el Gobierno de los Centros Docentes (LOPEG), promulgada en 1996, reforzó la necesidad de evaluar el sistema educativo, asentó el INCE y desarrolló más extensamente otros ámbitos de la acción evaluadora, como la evaluación de los centros o la valoración de elementos como la función pública docente, la función directiva y la inspección. Aunque la aplicación de la LOPEG tuvo un recorrido corto, a consecuencia del cambio de gobierno que se produjo ese mismo año, hay que reconocer que el título que dedicó a la evaluación dibujaba un cuadro bastante completo de un mecanismo integrado de evaluación del sistema educativo, en el que participarían organismos estatales y autonómicos. Era la primera vez en España que la evaluación del sistema educativo quedaba plenamente integrada en un texto legal con suficiente grado de detalle. El camino que la LGE apenas esbozó y la LOGSE continuó mediante el establecimiento de un marco normativo general, la LOPEG lo recorrería más decididamente, al trazar un cuadro de sus principales ámbitos y finalidades. Podemos decir que a mediados de los años noventa estaban bien establecidos los fundamentos institucionales de la evaluación general del sistema educativo español.

El cambio de gobierno de 1996 no supuso una quiebra en la evolución del modelo descrito. El INCE continuó existiendo y sus funciones siguieron siendo las mismas, si 
bien su plan de actuación se vio ligeramente modificado. Más adelante, la aprobación en diciembre de 2002 de la Ley Orgánica de Calidad de la Educación (LOCE) transformó el INCE en el Instituto Nacional de Evaluación y Calidad del Sistema Educativo (INECSE), a quien se continuó atribuyendo la evaluación general del sistema educativo.

La LOCE reforzó los planteamientos y la práctica de la evaluación del sistema educativo que venían desarrollándose durante la década anterior, dedicando a ese asunto un título completo, en el que se disponía que la evaluación se aplicará sobre "los procesos de aprendizaje de los alumnos, los procesos educativos, la actividad del profesorado, los centros docentes, la inspección de la educación y la propia Administración educativa" (artículo 93). La importancia concedida a la evaluación del sistema educativo era puesta en conexión en el preámbulo de la ley con la necesidad de "orientar más abiertamente el sistema educativo hacia los resultados" y con "la consolidación de la cultura del esfuerzo y la mejora de la calidad”. La evaluación era considerada por los promotores de la ley como "un factor básico de calidad", al mismo tiempo que "un instrumento ineludible para hacer inteligentes políticas educativas". El INECSE desarrollaría actividades semejantes a las del INCE, aunque el texto legal insistía especialmente en la responsabilidad que tendría en la realización de evaluaciones generales de diagnóstico "sobre áreas y asignaturas", que versarían sobre "competencias básicas del currículo" (artículo 95), lo que anticiparía algunas tendencias que se desarrollarían en la fase siguiente. El nuevo instituto debería continuar desarrollando el sistema estatal de indicadores de la educación ya iniciado y llevaría a cabo un plan de evaluación general del sistema educativo.

El INCE y el INECSE desarrollaron durante la década de 1994 a 2004 un buen número de estudios de evaluación del sistema educativo español o de algunos de sus niveles o componentes, de características bastante diversas ${ }^{3}$. Una buena parte de dichos estudios tuvieron carácter nacional, aunque también en esos años se incrementó notablemente la participación en estudios internacionales. Entre todos ellos, merece la pena señalar tres grandes grupos de proyectos.

\footnotetext{
${ }^{3}$ Las publicaciones de todos los trabajos realizados por el INCE, INECSE, IE e INEE, desde 1994 hasta la actualidad, pueden encontrarse y descargarse en

http://www.mecd.gob.es/inee/publicaciones/
} 
Un primer grupo importante de trabajos estuvo compuesto por diversos estudios de evaluación orientados a valorar empíricamente los resultados conseguidos por los estudiantes en determinadas áreas y etapas, por medio de la aplicación de pruebas de rendimiento y de cuestionarios a una muestra de centros, estudiantes, profesores y familias. Se enlazaba así con una tendencia claramente perceptible en los países desarrollados en la década de los noventa, que estaba marcando el sentido de las políticas de reforma educativa en lugares muy diversos (Tiana, 2003; Elmore, 2004).

Por medio de dichos trabajos se pretendía poner en marcha un proceso de evaluación permanente del sistema educativo. Con ese objetivo último se adoptó un plan periódico de evaluación de los resultados de la educación en las principales etapas educativas previas a la enseñanza universitaria, si bien los estudios realizados se circunscribieron básicamente a la Educación Primaria y la Educación Secundaria Obligatoria (ESO).

La Educación Primaria fue objeto de estudio en 1995, 1999, 2003 y 2007. Se construyeron y aplicaron pruebas de Conocimiento del medio, Lengua española y literatura y Matemáticas a una muestra amplia de estudiantes de $6^{\circ}$ curso y se prestó una especial atención a la posibilidad de comparar los resultados obtenidos a lo largo del tiempo. En 1995, la evaluación se llevó a cabo en el sexto curso de EGB (era el último año en que se impartía) y a partir de 1999 los alumnos fueron de sexto curso de Educación Primaria. Además, se llevaron a cabo algunos estudios singulares, con carácter experimental y no vinculados a los anteriores, en las áreas de educación física y lengua inglesa y en la Educación Infantil.

La ESO fue objeto de evaluación en 1997 y 2000. Las áreas evaluadas fueron en este caso la Lengua española y literatura, Matemáticas, Ciencias de la Naturaleza y Geografía e historia. En el año 1997 se tomaron datos de estudiantes de $4^{\circ}$ de ESO, pero también de $2^{\mathrm{O}}$ de BUP, $2^{\mathrm{O}}$ de Formación Profesional y $2^{\mathrm{O}}$ de la Reforma de las Enseñanzas Medias, etapas que llegaban por entonces a su extinción.

Un segundo grupo de trabajos estuvieron encaminados al desarrollo de un sistema estatal de indicadores de la educación. Siguiendo el ejemplo de países como Francia, que viene publicando anualmente desde 1991 su conjunto de indicadores titulado L'État de l’École, o de Bélgica, Suiza, Holanda o el Reino Unido, el INCE comenzó la elaboración de un sistema español similar a los anteriores. En el año 2000 se publicó su 
primera entrega, que ha continuado desarrollándose y depurándose desde entonces. Entre 2000 y 2008 se publicaron entregas sucesivas cada dos años y desde 2009 aparece una edición con carácter anual. Este trabajo se complementó con la participación activa en el Proyecto de Indicadores Internacionales de la Educación, promovido por la OCDE, que viene produciendo desde 1992 las publicaciones denominadas Education at a Glance / Regards sur l'éducation y que ha revolucionado el campo de la estadística educativa.

Un tercer grupo de trabajos se tradujo en la participación española en diversos proyectos internacionales. Aunque ya hemos visto que esa línea de actuación se remonta a los años anteriores a la creación del INCE, sería en esta etapa cuando experimentase un despegue importante.

En 1991 España participó en el estudio de comprensión lectora PIRLS, desarrollado por la IEA. En 1995 y 2003 lo hizo en el Tercer Estudio Internacional de Matemáticas y Ciencias (TIMSS), de la misma asociación. Y a partir del 2000 comenzó a participar en el Programme for International Student Assessment (PISA), impulsado por la OCDE, en el que continúa estando presente en la actualidad. Los dos tipos de proyectos, aunque tienen características ligeramente diferentes (los de la IEA se dirigen a los alumnos de $2^{\circ}$ de ESO, independientemente de su edad, mientras que los de la OCDE se dirigen a los jóvenes de 15 años, independientemente del nivel escolar en que se encuentren), coinciden en proporcionar datos de gran valor para situar a nuestro sistema educativo en el contexto internacional.

En conjunto, puede decirse que durante esta primera etapa analizada, que abarca de 1994 a 2004, asistimos a la constitución de un sistema de evaluación general del sistema educativo español. Dicha etapa se caracterizó por la articulación de un sistema de cooperación entre el Ministerio de Educación y las comunidades autónomas, cuyo fruto más patente fue la puesta en marcha de un amplio conjunto de proyectos de evaluación, de carácter nacional e internacional. Entre sus logros cabe señalar la puesta en marcha de varios estudios de evaluación de los resultados del aprendizaje de los estudiantes, en momentos clave de su escolaridad, de los que España prácticamente había carecido hasta entonces. Además, se vinculó a las organizaciones internacionales más activas en este campo, lo que permitió realizar un aprendizaje valioso para la puesta en marcha de los proyectos propios. Entre sus limitaciones, hay que mencionar 
la escasa difusión y utilización de sus informes en el debate público y en la construcción de políticas educativas, así como la inexistencia de análisis comparativos entre comunidades autónomas, que hubieran permitido conocer mejor las disparidades educativas que se dan en España. Por otra parte, hay que subrayar las dificultades del proceso de incorporación de las comunidades autónomas al sistema de evaluación, que tuvo su punto álgido en la retirada de algunas de ellas de los estudios emprendidos para realizar la evaluación de la ESO, como protesta a las actitudes ministeriales en ese momento. No obstante, cabe considerar que el proceso tuvo éxito en términos generales y que esta etapa sirvió para asentar un mecanismo estatal de evaluación general del sistema educativo.

\section{El desarrollo de las evaluaciones de diagnóstico de las competencias básicas}

El cambio de gobierno que se produjo en el año 2004 llevó consigo una reorientación de la política aplicada para llevar a cabo la evaluación general del sistema educativo. Aunque no puede decirse que se produjese una ruptura brusca en el programa que el INECSE venía desarrollando con anterioridad, se introdujeron algunos cambios significativos, que merece la pena destacar. Es así como se inició la segunda etapa de las que aquí se vienen considerando.

La transformación registrada tuvo que ver fundamentalmente con el carácter de las evaluaciones realizadas, que pasaron a concebirse no solo como un elemento de valoración del rendimiento de los estudiantes y del conjunto del sistema educativo, sino como un instrumento de diagnóstico, al servicio de la prevención de las dificultades de aprendizaje y de su tratamiento temprano. Dicho de otro modo, la evaluación no debería servir solamente para valorar el grado de logro obtenido por los estudiantes al final de cada etapa educativa, sino que habría de estar al servicio de la mejora de su rendimiento.

Este cambio de concepción estuvo ligado a una importante transformación curricular introducida en la educación básica por la Ley Orgánica de Educación (LOE) de 2006, consistente en la consideración de las competencias clave o básicas como un elemento constitutivo del currículo (Tiana, 2011; Tiana, Moya y Luengo, 2011). Este 
nuevo planteamiento venía siendo anticipado por organizaciones internacionales como la OCDE, que ya en los años noventa había centrado su atención en las habilidades o competencias desarrolladas por los estudiantes, más que en los conocimientos escolares. Proyectos como el denominado DeSeCo (Design and Selection of Competencies) anticiparon esa tendencia (Rychen y Salganik, 2001 y 2003), que a partir del año 2000 comenzaría a aplicar y desarrollar el conocido proyecto PISA.

Ese planteamiento de la OCDE fue posteriormente desarrollado por la Unión Europea, que aprobó la Recomendación del Parlamento Europeo y del Consejo de 18 de diciembre de 2006 sobre las competencias clave para el aprendizaje permanente (2006/962/CE). En ese documento las competencias se definen como "una combinación de conocimientos, capacidades y actitudes adecuadas al contexto". Las competencias clave, por su parte, son "aquéllas que todas las personas precisan para su realización y desarrollo personales, así como para la ciudadanía activa, la inclusión social y el empleo". Las competencias consideradas clave son las ocho siguientes: comunicación en la lengua materna; comunicación en lenguas extranjeras; competencia matemática y competencias básicas en ciencia y tecnología; competencia digital; aprender a aprender; competencias sociales y cívicas; sentido de la iniciativa y espíritu de empresa; y conciencia y expresión culturales.

Como cierre normativo de dicho planteamiento en España, el artículo 6 de la LOE definió el currículo como "el conjunto de objetivos, competencias básicas, contenidos, métodos pedagógicos y criterios de evaluación de cada una de las enseñanzas”. Se trata de una formulación similar a la que habían adoptado anteriormente tanto la LOGSE como la LOCE, pero introduciendo como novedad la referencia expresa a las competencias básicas que, de ese modo, entraban a formar parte de los currículos de la educación española. Una vez incluidas de forma genérica en la definición del currículo, las competencias básicas fueron utilizadas como referencia expresa en otros artículos de la ley, fundamentalmente en relación con la evaluación y promoción del alumnado (artículos 20 y 28), las evaluaciones de diagnóstico (artículos 21, 29 y 144) y la titulación al final de la ESO (artículo 31). La decisión era coherente puesto que, si se considera que las competencias básicas representan los saberes considerados imprescindibles para todos los jóvenes, deben servir como referencia para evaluar los resultados educativos alcanzados y tomarse en consideración para decidir aspectos tan relevantes como la promoción de etapa y la titulación de los estudiantes. 
Esta transformación introducida en la concepción y la definición del currículo tuvo un efecto directo sobre la organización de la evaluación general del sistema educativo. Si la evaluación debía pasar a concebirse como un elemento de diagnóstico, cumpliendo una función pedagógica formativa y no solamente sumativa o de juicio de los resultados finales de una determinada etapa, convenía revisar el momento en que las pruebas generales debían llevarse a cabo. La decisión tomada por la LOE consistió en situarlas al finalizar el cuarto curso de la Educación Primaria y el segundo de la ESO. Evaluar el avance registrado en esos puntos escolares ofrecería la posibilidad de actuar en lo dos años siguientes para reorientar el proceso de enseñanza y aprendizaje y mejorar así el rendimiento al final de la etapa correspondiente. Por lo tanto, las nuevas evaluaciones generales de diagnóstico deberían realizarse en los cursos mencionados.

De forma paralela, las pruebas aplicadas deberían tender a evaluar las competencias desarrolladas por los estudiantes, más que a medir sus conocimientos escolares. Se trataba de un planteamiento novedoso, similar al que la OCDE aplica en el proyecto PISA y que actualmente despierta el interés de muchos evaluadores de la educación, como han puesto recientemente de relieve algunos especialistas (Roca, 2013).

De acuerdo con ese planteamiento general, el Instituto de Evaluación (nueva denominación que dio la LOE al anterior INECSE) puso en marcha el desarrollo de las evaluaciones de diagnóstico, que se aplicaron por primera vez en los años 2009 (cuarto curso de Educación Primaria) y 2010 (segundo curso de ESO). Dicha tarea se complementó con la llevada a cabo por los organismos autonómicos de evaluación, que en muchos casos también desarrollaron sus propias pruebas de diagnóstico. Es así como se produjo un giro interesante en el modelo de evaluación general del sistema educativo español que, sin embargo, no tuvo larga vida, como veremos.

Si la evaluación diagnóstica de las competencias básicas supuso el eje central de las actividades desarrolladas en esta segunda etapa, no agotó el plan de actuación del Instituto de Evaluación. También se puso en marcha una segunda línea de trabajo, ciertamente fructífera y que continúa vigente en la actualidad, que engarza directamente con el seguimiento de los objetivos educativos establecidos por la Unión Europea para 2010 y posteriormente para 2020. 
Efectivamente, la conciencia de la necesidad de mejorar los resultados educativos españoles llevó a establecer un mecanismo de seguimiento del cumplimiento por parte de España de los objetivos mencionados, que permitiese al mismo tiempo su mejora paulatina. Con ese propósito, la Conferencia sectorial de Educación discutió en mayo de 2005 una propuesta de identificación de diez objetivos a conseguir para 2010, con sus respectivos puntos de referencia debidamente temporalizados. Seis de ellos eran objetivos establecidos a nivel europeo4, mientras que los cuatro restantes eran específicamente españoles5. Como fruto de ese acuerdo, la Comisión general de Educación recibió en enero de 2006 un primer informe de situación y decidió crear dos grupos de trabajo con el propósito de analizar y proponer posibles medidas de mejora. Tanto el informe inicial como las principales propuestas de mejora y un análisis de las medidas ya adoptadas fueron objeto de una interesante publicación al año siguiente (MEC, 2007). Conviene destacar que estos objetivos y sus correspondientes puntos de referencia fueron la base para la confección de la parte del Plan nacional de reformas relativa al desarrollo de los recursos humanos.

Esta línea de trabajo se ha mantenido hasta la actualidad, produciendo informes anuales de gran interés. Además, coexiste con la publicación del sistema nacional de indicadores de la evaluación, al que se hizo referencia en el epígrafe anterior. La combinación de ambas fuentes aporta una información ciertamente valiosa para analizar y valorar la situación y la evolución del sistema educativo español, desde una perspectiva general y macroscópica.

Junto a estas dos líneas de trabajo, el Instituto de Evaluación continuó sosteniendo la participación española en diversos estudios internacionales de evaluación. Además de PISA, que viene desarrollándose desde 2010 y recibe una gran atención mediática, en esta segunda etapa España ha participado, entre otros proyectos, en los estudios denominados Teaching and Learning International Study

\footnotetext{
${ }^{4}$ Se trata de los siguientes objetivos: reducir el abandono escolar prematuro; mejorar el rendimiento de los alumnos en competencias clave; incrementar la proporción de alumnado titulado en educación secundaria superior; aumentar la tasa de alumnos graduados en ciencias, matemáticas y tecnología; incrementar el porcentaje de adultos que recibe formación permanente; incrementar el gasto público en educación en relación con el PIB.

5 Se trata de los siguientes objetivos: incrementar la tasa de escolarización en educación infantil; incrementar la tasa de alumnos titulados en ESO; incrementar la tasa de varones titulados en los estudios de educación secundaria postobligatoria; incrementar el porcentaje de graduados en formación profesional de grado superior.
} 
(TALIS), realizado en 2008 por la OCDE; Progress in Reading Literacy Study (PIRLS) y Third International Mathematics and Science Study (TIMSS), desarrollados por la IEA, en 2006 y 2011 en el primer caso, y en 2011 en el segundo; o en el International Civic and Citizenship Education Study (ICCS), realizado por la IEA en 20096.

En conjunto, esta segunda fase supuso una reorientación de la política de evaluación general del sistema educativo que se había iniciado en los años noventa. En todo caso, no representó un giro abrupto, ni una ruptura con la línea anterior, pues muchas de sus actuaciones mantuvieron una lógica continuidad. Pero el cambio de foco que se intentó llevar a cabo en lo relativo a la evaluación del rendimiento de los estudiantes merece la pena ser destacado.

\section{El predominio de las evaluaciones individualizadas}

La aprobación de la Ley Orgánica de Mejora de la Calidad de la Educación (LOMCE) en 2013 ha supuesto el inicio de una nueva etapa en la política de evaluación general del sistema educativo español. Además de cambiar nuevamente el nombre del organismo responsable de llevarla a cabo, que ha pasado a denominarse Instituto Nacional de Evaluación Educativa (INEE), el elemento más destacado del cambio de modelo consiste en la desaparición de las evaluaciones de diagnóstico que introdujo la LOE. El símbolo más visible de ese cambio se encuentra en la sustitución del artículo 144 de la LOE, titulado "Evaluaciones generales de diagnóstico", por uno nuevo, titulado "Evaluaciones individualizadas".

Con este nombre la LOMCE se refiere a todas las nuevas evaluaciones finales de etapa que habrán de pasar los estudiantes de Educación Primaria, ESO y Bachillerato. La verdad es que, aunque se denominen en la ley evaluaciones finales o individualizadas, en realidad se trata de reválidas, similares a las que suprimió la Ley General de Educación (LGE) de 1970. Y dichas reválidas serán a partir de ahora la base de la evaluación general del sistema educativo, como da a entender el cambio producido en el artículo mencionado.

\footnotetext{
6 Se puede encontrar información más completa sobre estos y otros estudios internacionales con participación española en http://www.mecd.gob.es/inee/publicaciones/estudios-internacionales.html.
} 
Estas nuevas pruebas tendrán al mismo tiempo carácter individual, puesto que determinarán la promoción de etapa y la titulación de los estudiantes, individualmente considerados, y general, puesto que proporcionarán información sobre la situación del conjunto del sistema educativo. Las pruebas prestarán también una atención especial a la adquisición de competencias básicas por parte de los estudiantes, lo que supone un cierto punto de continuidad con la etapa anterior.

Se trata de un mecanismo complejo, puesto que, si se quiere evaluar con justicia a cada estudiante, las pruebas habrán de contemplar todos los objetivos curriculares relevantes de cada etapa. Pero, al mismo tiempo, la realización de evaluaciones estandarizadas capaces de proporcionar una información relevante acerca del conjunto del sistema educativo plantea unas exigencias metodológicas propias, que llevan, por ejemplo, a la aplicación de cuadernillos combinados, distintos para cada estudiante, que impiden obtener puntuaciones individuales comparables. En consecuencia, es previsible que se plantee una opción complicada o que se limite la potencia evaluativa de dichas pruebas para una u otra finalidad. Pero es cierto que el modo en que ese modelo se desarrolle está aún por ver y habrá que esperar para valorarlo adecuadamente.

$\mathrm{Al}$ margen de este cambio fundamental, no son de esperar otros giros en el plan de actuación del INEE. La continuidad de la elaboración y publicación del sistema estatal de indicadores educativos y la participación en estudios internacionales está asegurada, como parece lógico en la situación actual. Y también la participación activa, e incluso reforzada, en las tareas de seguimiento de los objetivos educativos europeos y españoles está fuera de discusión. Por lo tanto, al igual que ha sucedido en las dos etapas anteriores, pueden identificarse algunas continuidades, junto a claras rupturas.

Es difícil aventurar cómo evolucionarán exactamente estos planteamientos, pues aún se encuentran en sus inicios. En todo caso, no cabe duda de que el cambio de foco que se ha producido en la evaluación nos permite hablar de una tercera etapa en el proceso que venimos analizando. 


\section{A modo de conclusión}

Como ha podido comprobarse, a lo largo de estas dos últimas décadas se ha ido configurando un sistema de evaluación general del sistema educativo, que está plenamente activo en la actualidad. Lo que empezó como una serie de tanteos en la década de los ochenta, es hoy un sistema bien asentado y consolidado. Hoy podemos decir que, al igual que la gran mayoría de los países desarrollados, España cuenta con experiencia, proyectos y presencia suficientes en el ámbito de la evaluación de los sistemas educativos.

En el proceso de construcción de dicho sistema se pueden distinguir tres etapas. Cada una de ellas tiene unos rasgos distintivos, que han sido expuestos y analizados en sucesivos epígrafes. La primera se centró en la aplicación de pruebas de resultados curriculares a muestras representativas de estudiantes, la segunda en la elaboración de pruebas de diagnóstico de las competencias básicas adquiridas y la tercera lo estará en la realización de pruebas individualizadas. Se trata de enfoques diferentes, aunque algunos de sus rasgos presentan elementos de continuidad. Por otra parte, son varios los proyectos que han venido desarrollándose de manera continuada a través de las etapas identificadas.

El sistema de evaluación general del sistema educativo construido y desarrollado a lo largo de estas dos décadas presenta, por tanto, continuidades y rupturas. Hay unas líneas de trabajo sostenidas en el tiempo, que tienen que ver sobre todo con la construcción de indicadores de la educación y con la participación creciente en estudios internacionales. Y hay también elementos que han ido cambiando, referidos sobre todo al objeto de la evaluación - contenidos curriculares o competencias básicas -, a su propósito - sumativo o formativo - y a la ubicación de las pruebas - al final o no de las etapas educativas. Pero, en conjunto, lo que se puede afirmar es que estos veinte años han visto diseñarse, organizarse y desarrollarse un sistema de evaluación general del sistema educativo, que aún debe dar muchos más frutos en el futuro. 


\section{Referencias}

CIDE (1988). Evaluación Externa de la Reforma Experimental de las Enseñanzas Medias (I). Madrid: Ministerio de Educación y Ciencia - Centro de Investigación, Documentación y Evaluación.

CIDE (1990). Evaluación Externa de la Reforma Experimental de las Enseñanzas Medias (II). Madrid: Ministerio de Educación y Ciencia - Centro de Investigación, Documentación y Evaluación.

CIDE (1992). Evaluación Externa de la Reforma Experimental de las Enseñanzas Medias (III). Madrid: Ministerio de Educación y Ciencia - Centro de Investigación, Documentación y Evaluación.

Elmore, R.F. (2004). School Reform from the Inside Out. Policy, Practices, and Performance. Camdridge, Mass.: Harvard Education Press.

García Sánchez, E. (2011). Evaluación de políticas y reformas educativas en España (1982-1992). Tres experiencias de metaevaluación. Madrid: Instituto Nacional de Administración Pública.

MEC (1969). La educación en España. Bases para una política educativa. Madrid: Ministerio de Educación y Ciencia.

MEC (1986). Examen de la política educativa española por la OCDE. Madrid: Ministerio de Educación y Ciencia-CIDE.

MEC (1987). Proyecto para la reforma de la enseñanza. Propuesta para debate. Madrid: Ministerio de Educación y Ciencia.

MEC (1989). Libro Blanco para la Reforma del Sistema Educativo. Madrid: Ministerio de Educación y Ciencia.

MEC (2007). Informe 2006: Objetivos Educativos y Puntos de Referencia 2010. Madrid: Ministerio de Educación y Ciencia - Secretaría General de Educación.

Menor, M. y Moreiro, J., Coords. (2010). La reforma experimental de las enseñanzas medias (1983-1987). Crónica de una ilusión. Las Rozas (Madrid): Wolters Kluwer.

Pérez, A. y Gimeno, J. (1994). Evaluación de un proceso de innovación educativa. Sevilla: Junta de Andalucía - Consejería de Educación y Ciencia.

Roca, E. (2013). La evaluación diagnóstica de las competencias básicas. Madrid: Síntesis.

Rychen, D.S. y Salganik, L.H. (eds.) (2001). Defining and selecting key competencies. Seattle, Toronto, Bern \& Göttingen: Hogrefe and Huber. 
Rychen, D.S. y Salganik, L.H. (eds.) (2003). Key Competencies for a Successful Life and a Well-Functioning Society. Seattle, Toronto, Bern \& Göttingen: Hogrefe and Huber.

Tiana, A. (2003). Pilotage par les résultats et amélioration de l'éducation: deux réalités indissociables?, Administration et Éducation, $\mathrm{n}^{\circ}$ 98, pp. 19-29.

Tiana, A. (2011). Análisis de las competencias básicas como núcleo curricular en la educación obligatoria española, Bordón, vol. 63, nº 1, pp. 63-75.

Tiana, A.; Moya, J. y Luengo, F. (2011) Developing and Implementing Key Competences in Basic Education: analyses and reflections on curriculum design and development from the Spanish experience, European Journal of Education, vol. $46, \mathrm{n}^{\mathrm{o}} 3$, pp. 307-322.

Varios autores (2007). 10 años de evaluación de la calidad de las universidades (19962006). Madrid: ANECA. 